\title{
Atração miraxonal exercida por Biomphalaria straminea, Lymnaea columella e Physa sp, sobre miracídios de Schistosoma mansoni da linhagem $\mathrm{BH}^{*}$
}

\author{
Luiz A. Magalhães", Eliana M. Zanotti-Magalhães"**, José F. de Carvalho ***, Marina Faraone****
}

\begin{abstract}
MAGALHÃES, L. A. et al. Atração miraxonal exercida por Biomphalaria straminea, Lymnaea columella e Physa sp, sobre miracídios de Schistosoma mansoni da linhagem BH. Rev. Saúde públ., S. Paulo, 25: 23-7, 1991. Utilizando-se um artefato de vidro composto de duas câmaras unidas por um canal, testamos a atração miraxonal exercida por Biomphalaria straminea, Lymnaea columella e Physa sp, sobre larvas de Schistosoma mansoni da linhagem BH. Os moluscos, ou suas águas de condicionamento ( $\mathrm{SCW}$ ), foram colocados aleatoriamente em uma das câmaras, contendo o restante do artefato somente água declorada. Dez miracídios foram depositados no centro do canal. $\mathrm{O}$ comportamento dos miracídios foi observado por $15 \mathrm{~min}$. Foram feitas dez replicações de cada experimento, utilizando-se espécimens de moluscos e miracídios diferentes. Verificou-se que todos os moluscos e suas SCW exerceram atração miraxonal, sendo que Physa sp atraiu mais que Lymnaea columella. Foi isolado um único exemplar de $B$. straminea que exerceu efeito repulsivo sobre os miracídios de $S$. mansoni.
\end{abstract}

Descritores: Biomphalaria, fisiologia. Lymnaea, fisiologia. Schistosoma mansoni, fisiologia. Atividade motora.

\section{Introduçăo}

Após os trabalhos de Kloetzel ${ }^{4,5}(1958,1960)$, Etges e col. ${ }^{6}$ (1963) e Brasio e col. ${ }^{1}$ (1985), ficou confirmada a existência de atração exercida por moluscos vetores sobre miracídios de Schistosoma mansoni.

Foi verificado que os moluscos Helisoma anceps e Bulinus sp, não vetores de $S$. mansoni (Etges e col. $\left.{ }^{6}, 1963\right)$, e girinos de Hyla fuscovaria (Brasio e col. ${ }^{1}$, 1985) não exerciam atração miraxonal sobre larvas deste trematódeo.

A atração do miracídio exercida pelos hospedeiros intermediários faz-se mediante a ação de substâncias emanadas pelo caramujo na água. A água contendo essas substâncias, convencionou-se chamar de SCW, "snail conditioned water" (Chernin $^{2}, 1972$ ). Brasio e col. ${ }^{1}$ (1985) constataram a ação miraxonal de várias substâncias contidas na SCW e na hemolinfa de moluscos planorbídeos.

* Realizado com auxílio financeiro do Conselho Nacional de Desenvolvimento Científico e Tecnológico ( $\mathrm{CNPq}$ Processo n 405937/88).

* Departamento de Parasitologia do Instituto de Biologia da Universidade Estadual de Campinas (UNICAMP) - Caixa Postal 6109 - 13081 Campinas, SP - Brasil. Pesquisadores do CNPq.

*** Departamento de Estatística do Instituto de Matemática e Ciências da Computação da UNICAMP.

**** Departamento de Parasitologia da UNICAMP Estagiária.
No presente trabalho pesquisamos a atração miraxonal exercida por Biomphalaria straminea e por sua SCW sobre miracídios de $S$. mansoni da linhagem BH (Paraense e col. ${ }^{8}, 1963$ ). Testamos também a possível atração miraxonal de Lymnaea columella e Physa sp (moluscos não vetores de $S$. mansoni) e de suas SCW sobre miracídios do mesmo trematódeo.

A pesquisa de atração miraxonal em moluscos não vetores do $S$. mansoni tem interesse em saúde pública, uma vez que os miracídios deste trematódeo poderão ser desviados de seus hospedeiros invertebrados em focos onde subsistam espécies de moluscos resistentes à infeç̧ão.

\section{Material e Método}

Os moluscos utilizados no experimento foram exemplares adultos obtidos do moluscário do Departamento de Parasitologia da Universidade Estadual de Campinas (UNICAMP). Os exemplares de $B$. straminea eram descendentes de moluscos capturados no campo em Picos, Piauí. Os exemplares de $L$. columella e $P$ hysa sp eram descendentes de moluscos capturados na regiāo de Campinas, SP.

Para a observação do comportamento dos miracídios, utilizou-se um artefato de vidro composto de duas câmaras circulares com $30 \mathrm{~mm}$ de diâmetro e $20 \mathrm{~mm}$ de profundidade, unidas por um canal de $40 \mathrm{~mm}$ de comprimento, $11 \mathrm{~mm}$ de largura e $10 \mathrm{~mm}$ de profundidade (Brasio e col. ${ }^{1}$, 1985). Os experimentos foram realizados colo- 
Tabela 1. Número de miracidios de $S$. mansoni nas cámaras $A$ (molusco) e $B$ (água) e no canal $C$ (contendo 10 miracídios) após 15 min de observaçăo, quando expostos a açăo de uma das espécies de moluscos: $B$. straminea, Lymnaea columella ou Physa sp, na luz e na penumbra.

\begin{tabular}{|c|c|c|c|c|c|c|c|c|c|c|c|c|c|c|c|c|c|}
\hline \multicolumn{6}{|c|}{ B. straminea } & \multicolumn{6}{|c|}{ Lymnaea columella } & \multicolumn{6}{|c|}{ Physa sp } \\
\hline \multicolumn{3}{|c|}{ luz } & \multicolumn{3}{|c|}{ penum. } & \multicolumn{3}{|c|}{ luz } & \multicolumn{3}{|c|}{ penum. } & \multicolumn{3}{|c|}{ luz } & \multicolumn{3}{|c|}{ penum. } \\
\hline A & C & B & A & $c$ & B & $A$ & C & B & A & c & B & A & c & B & A & c & B \\
\hline $\begin{array}{r}8 \\
9 \\
10 \\
9 \\
9 \\
7 \\
6 \\
8 \\
7 \\
7\end{array}$ & $\begin{array}{l}1 \\
0 \\
0 \\
0 \\
0 \\
0 \\
0 \\
1 \\
0 \\
0\end{array}$ & $\begin{array}{l}1 \\
1 \\
0 \\
1 \\
1 \\
3 \\
4 \\
1 \\
3 \\
3\end{array}$ & $\begin{array}{r}10 \\
7 \\
7 \\
9 \\
8 \\
9 \\
10 \\
8 \\
8 \\
8\end{array}$ & $\begin{array}{l}0 \\
2 \\
1 \\
1 \\
1 \\
0 \\
0 \\
0 \\
0 \\
1\end{array}$ & $\begin{array}{l}0 \\
1 \\
2 \\
0 \\
1 \\
1 \\
0 \\
2 \\
2 \\
1\end{array}$ & $\begin{array}{l}7 \\
8 \\
7 \\
6 \\
5 \\
6 \\
6 \\
8 \\
6 \\
6\end{array}$ & $\begin{array}{l}1 \\
0 \\
1 \\
0 \\
0 \\
0 \\
0 \\
0 \\
0 \\
0\end{array}$ & $\begin{array}{l}2 \\
2 \\
2 \\
4 \\
5 \\
4 \\
4 \\
2 \\
4 \\
4\end{array}$ & $\begin{array}{l}8 \\
6 \\
7 \\
7 \\
7 \\
9 \\
9 \\
8 \\
5 \\
5\end{array}$ & $\begin{array}{l}0 \\
1 \\
0 \\
0 \\
0 \\
0 \\
0 \\
0 \\
0 \\
0\end{array}$ & $\begin{array}{l}2 \\
3 \\
3 \\
3 \\
3 \\
1 \\
1 \\
2 \\
5 \\
5\end{array}$ & $\begin{array}{l}8 \\
9 \\
8 \\
8 \\
7 \\
8 \\
6 \\
7 \\
8 \\
8\end{array}$ & $\begin{array}{l}0 \\
0 \\
0 \\
0 \\
0 \\
0 \\
0 \\
0 \\
0 \\
0\end{array}$ & $\begin{array}{l}2 \\
1 \\
2 \\
2 \\
3 \\
2 \\
4 \\
3 \\
2 \\
2\end{array}$ & $\begin{array}{r}10 \\
9 \\
9 \\
7 \\
8 \\
10 \\
8 \\
9 \\
10 \\
8\end{array}$ & $\begin{array}{l}0 \\
0 \\
0 \\
0 \\
0 \\
0 \\
1 \\
0 \\
0 \\
0\end{array}$ & $\begin{array}{l}0 \\
1 \\
1 \\
3 \\
2 \\
0 \\
1 \\
1 \\
0 \\
2\end{array}$ \\
\hline
\end{tabular}

Tabela 2. Número de miracidios de $S$. mansoni nas câmaras $A$ (SCW) e $B$ (água) e no canal $C$ (contendo 10 miracídios) após 15 minutos de observaçáo, quando expostos a ação da SCW de uma das espécies de moluscos: $B$. straminea, Lymnaea columella ou Physa sp, na luz e na penumbra.

\begin{tabular}{|c|c|c|c|c|c|c|c|c|c|c|c|c|c|c|c|c|c|}
\hline \multicolumn{6}{|c|}{ B. straminea } & \multicolumn{6}{|c|}{ Lymnaea columella } & \multicolumn{6}{|c|}{ Physasp } \\
\hline \multicolumn{3}{|c|}{ luz } & \multicolumn{3}{|c|}{ penum. } & \multicolumn{3}{|c|}{ luz } & \multicolumn{3}{|c|}{ penum. } & \multicolumn{3}{|c|}{ Iuz } & \multicolumn{3}{|c|}{ penum. } \\
\hline A & $c$ & B & A & $c$ & $\mathbf{B}$ & A & $c$ & B & A & c & $B$ & A & c & B & A & c & B \\
\hline $\begin{array}{l}9 \\
8 \\
7 \\
8 \\
8 \\
8 \\
7 \\
9 \\
8 \\
7\end{array}$ & $\begin{array}{l}1 \\
2 \\
2 \\
0 \\
1 \\
2 \\
1 \\
0 \\
2 \\
3\end{array}$ & $\begin{array}{l}0 \\
0 \\
1 \\
2 \\
1 \\
0 \\
2 \\
1 \\
0 \\
0\end{array}$ & $\begin{array}{r}9 \\
9 \\
9 \\
10 \\
7 \\
8 \\
7 \\
9 \\
8 \\
7\end{array}$ & $\begin{array}{l}0 \\
0 \\
1 \\
0 \\
3 \\
0 \\
0 \\
0 \\
1 \\
1\end{array}$ & $\begin{array}{l}1 \\
1 \\
0 \\
0 \\
0 \\
2 \\
3 \\
1 \\
1 \\
2\end{array}$ & $\begin{array}{l}6 \\
7 \\
6 \\
6 \\
6 \\
7 \\
7 \\
7 \\
7 \\
6\end{array}$ & $\begin{array}{l}0 \\
0 \\
2 \\
3 \\
2 \\
0 \\
0 \\
1 \\
1 \\
0\end{array}$ & $\begin{array}{l}4 \\
3 \\
2 \\
1 \\
2 \\
3 \\
3 \\
2 \\
4 \\
4\end{array}$ & $\begin{array}{l}7 \\
6 \\
6 \\
6 \\
7 \\
6 \\
7 \\
6 \\
8 \\
7\end{array}$ & $\begin{array}{l}3 \\
2 \\
0 \\
2 \\
1 \\
0 \\
0 \\
3 \\
1 \\
2\end{array}$ & $\begin{array}{l}0 \\
2 \\
4 \\
2 \\
2 \\
4 \\
3 \\
1 \\
1 \\
1\end{array}$ & $\begin{array}{l}8 \\
9 \\
8 \\
8 \\
7 \\
6 \\
7 \\
8 \\
9 \\
8\end{array}$ & $\begin{array}{l}2 \\
0 \\
0 \\
2 \\
0 \\
1 \\
3 \\
0 \\
1 \\
2\end{array}$ & $\begin{array}{l}0 \\
1 \\
2 \\
0 \\
3 \\
3 \\
0 \\
2 \\
0 \\
0\end{array}$ & $\begin{array}{r}8 \\
9 \\
8 \\
8 \\
7 \\
9 \\
10 \\
8 \\
7 \\
7\end{array}$ & $\begin{array}{l}0 \\
1 \\
0 \\
1 \\
2 \\
1 \\
0 \\
2 \\
2 \\
2\end{array}$ & $\begin{array}{l}2 \\
0 \\
2 \\
1 \\
1 \\
0 \\
0 \\
0 \\
1 \\
1\end{array}$ \\
\hline
\end{tabular}

cando-se um molusco ou a SCW correspondente, aleatoriamente, em uma das câmaras. A SCW foi obtida de um "pool" de moluscos, segundo Cher$\operatorname{nin}^{2}$ (1972). Os miracídios de S. mansoni da linhagem $\mathrm{BH}$, em número de 10 , foram depositados no centro do canal. A outra câmara foi preenchida com água declorada. Durante $15 \mathrm{~min}$. observou-se o comportamento das larvas em lupa estereoscópica com lente frontal redutora (X 0.5 ). $O$ experimento foi repetido 10 vezes com iluminação abundante, homogeneamente dispersa e, em número igual de vezes, na penumbra. Em cada replicação foram utilizados diferentes espécimens de moluscos e de larvas. A fim de comprovar a neutralidade da água utilizada quanto ao aspecto de atração miraxonal e também testar a eficiência do artefato, verificamos o comportamento dos miracídios quando as duas câmaras continham somente água declorada.
Utilizamos para análise o modelo de regressão logística multivariada devido a $\operatorname{Cox}^{3}$ (1970). Com o modelo, buscamos estudar as probabilidades $p$ (A), p (B) e p (C) das larvas passarem a câmara A ou $B$, ou permanecerem no canal. Cada combinação dos fatores estudados: iluminação, tipo (molusco ou SCW) e espécie ( $L$. columella, Physa sp ou $B$. straminea) forma uma população, com valores dessas probabilidades, que devem ser comparadas entre as populaçð̃es.

Os cálculos foram feitos com o sistema SAS, em micro-computador compatível com IBM PCXT, sob DOS 3.2.

\section{Resultados}

Os dados numéricos do experimento estão contidos nas Tabelas de 1 a 5 . 
Tabela 3. Número de miracídios de $S$. mansoni presentes nas câmaras A e B e no canal C, após 15 min de observaçăo, quando expostos simultaneamente a açăo do molusco Lymnaea columella (câmara A) e Physa sp (cámara B) ou as suas SCW, na luz e na penumbra.

\begin{tabular}{|c|c|c|c|c|c|c|c|c|c|c|c|}
\hline \multicolumn{4}{|c|}{ Lymnaea columella (A) } & \multicolumn{4}{|c|}{ Canal (C) } & \multicolumn{4}{|c|}{ Physa sp (B) } \\
\hline \multicolumn{2}{|c|}{ Molusco } & \multicolumn{2}{|c|}{ SCW } & \multicolumn{2}{|c|}{ Molusco } & \multicolumn{2}{|c|}{ SCW } & \multicolumn{2}{|c|}{ Molusco } & \multicolumn{2}{|c|}{ SCW } \\
\hline luz & penum. & luz & penum. & luz & penum. & Iuz & penum. & luz & penum. & uz & penum. \\
\hline 2 & 1 & 1 & 1 & 0 & 2 & 1 & 0 & 8 & 7 & 8 & 9 \\
\hline 5 & 1 & 3 & 1 & 0 & 1 & 0 & 3 & 5 & 8 & 7 & 6 \\
\hline 2 & 0 & 3 & 2 & 0 & 1 & 0 & 0 & 8 & 9 & 7 & 8 \\
\hline 2 & 0 & 1 & 2 & 0 & 0 & 2 & 0 & 8 & 10 & 7 & 8 \\
\hline 1 & 0 & 0 & 3 & 0 & 1 & 1 & 0 & 9 & 9 & 9 & 7 \\
\hline 2 & 2 & 1 & 0 & 0 & 0 & 1 & 1 & 8 & 8 & 8 & 9 \\
\hline 1 & 3 & 0 & 2 & 0 & 0 & 1 & 1 & 9 & 7 & 9 & 7 \\
\hline 3 & 3 & 2 & 0 & 0 & 0 & 1 & 1 & 7 & 7 & 7 & 9 \\
\hline 2 & 2 & 1 & 3 & 0 & 1 & 3 & 0 & 8 & 7 & 6 & 7 \\
\hline 3 & 0 & 0 & 1 & 0 & 1 & 1 & 1 & 7 & 9 & 9 & 9 \\
\hline
\end{tabular}

Tabela 4. Número de miracidios de S. mansoni presentes nas câmaras $A$ e $B$ e no canal $C$, após $15 \mathrm{~min}$ de observação, quando todo o aparelho continha somente água declorada, na luz e na penumbra.

\begin{tabular}{rrrrrr}
\hline \multicolumn{2}{r}{ Câmara A } & \multicolumn{2}{c}{ Canal C } & \multicolumn{2}{c}{ Câmara B } \\
\hline luz & penum. & luz & penum. & kz & penum. \\
\hline 2 & 0 & 7 & 9 & 1 & 1 \\
1 & 0 & 6 & 10 & 3 & 0 \\
1 & 1 & 7 & 8 & 2 & 1 \\
1 & 0 & 8 & 10 & 1 & 0 \\
1 & 2 & 6 & 7 & 3 & 1 \\
1 & 0 & 9 & 10 & 0 & 0 \\
3 & 0 & 7 & 9 & 0 & 1 \\
2 & 0 & 7 & 9 & 1 & 1 \\
0 & 0 & 9 & 9 & 1 & 1 \\
0 & 1 & 8 & 9 & 2 & 0 \\
\hline
\end{tabular}

No decorrer do experimento com exemplares de $B$. straminea, observou-se que um exemplar exercia uma ação repulsiva sobre os miracídios. Em cerca de 3 min. todos os miracídios dirigiram-se a câmara oposta. Este exemplar foi então separado para a realização de um experimento isolado. Foram feitas 10 observações com o mesmo molusco, e com mi-
Tabela 5. Número de miracídios de $S$. mansoni presentes nas câmaras $A$ e $B$ e no canal $C$, após 15 min de observação, quando expostos a ação de um único exemplar de B. straminea refratário (câmara A). Experimento realizado na luz.

\begin{tabular}{ccc}
\hline B. straminea $(A)$ & Canal C & Câmara B \\
\hline 0 & 0 & 10 \\
0 & 1 & 9 \\
1 & 1 & 8 \\
0 & 1 & 9 \\
0 & 2 & 8 \\
2 & 0 & 8 \\
2 & 0 & 8 \\
2 & 0 & 8 \\
2 & 1 & 7 \\
1 & 2 & 7 \\
\hline
\end{tabular}

racídios diferentes, sob a luz (Tabela 5). O exemplar morreu dias após a realização do experimento, não tendo sido possivel obter descendentes.

A Tabela 6 mostra que não houve interação entre os fatores, isto é, eventuais diferenças entre as probabilidades, se existirem, são constantes para quaisquer pares populacionais. Além disso, 
Tabela 6. Estudo da interação entre os fatores.

\begin{tabular}{lccc}
\hline \multicolumn{1}{c}{ Fonte } & GL & Qui-quadrado & Prob. \\
\hline Intercept & 2 & 328,10 & 0,0000 \\
Molusco & 4 & 22,94 & 0,0001 \\
Tipo & 2 & 3,78 & 0,1513 \\
Molusco Tipo & 4 & 0,30 & 0,9901 \\
Luz & 2 & 3,61 & 0,1643 \\
Molusco Luz & 4 & 1,07 & 0,8991 \\
Tipo Luz & 2 & 4,23 & 0,1206 \\
Molusco Tipo Luz & 4 & 1,49 & 0,8277 \\
\hline
\end{tabular}

Tabela 7. Comparação entre as câmaras A e B.

\begin{tabular}{|c|c|c|c|c|c|}
\hline Efeito & $\begin{array}{l}\text { Esti- } \\
\text { mativa }\end{array}$ & Param. & $\begin{array}{l}\text { Desvio } \\
\text { Padrão }\end{array}$ & $\begin{array}{l}\text { Qui-qua } \\
\text { drado. }\end{array}$ & Prob. \\
\hline Intercept & 1 & 0,4476 & 0,0209 & 458,16 & 0,0000 \\
\hline Luz & 2 & $-0,0333$ & 0,0208 & 2,57 & 0,1086 \\
\hline Tipo & 3 & 0,0275 & 0,0207 & 1,76 & 0,1852 \\
\hline \multirow[t]{2}{*}{ Molusco } & 4 & $-0,1083$ & 0,0311 & 12,14 & 0,0005 \\
\hline & 5 & 0,0305 & 0,0291 & 1,10 & 0,2946 \\
\hline
\end{tabular}

dos efeitos principais apenas o Molusco revelou-se significativo: existe diferença entre as probabilidades por espécies de moluscos. Não houve diferença na atração entre o molusco e sua SCW. A iluminação tampouco teve efeito.

Finalmente, comparando-se apenas as câmaras A e B (deixando-se o canal C), para ver se, realmente $\mathrm{P}(\mathrm{A})>\mathrm{P}(\mathrm{B})$, obtivemos o resultado constante da Tabela 7.

Vê-se que apenas a primeira comparação de molusco foi significativa $(\mathrm{p}=0.0005)$ : esta corresponde a $L$. columella versus $B$. straminea. Não há diferença na atração entre $B$. straminea e Physa sp.

\section{Discussão e Conclusóes}

Ao contrário de Etges e col. ${ }^{6}$ (1963), verificouse que moluscos não vetores de $S$. mansoni (L. Columella e Physa sp) atraíram miracídios deste trematódeo. A presença de moluscos não vetores, que atraem miracidios de $S$. mansoni em um foco, é uma condição que certamente interferirá no ciclo do trematódeo. Os miracídios poderão ser atraídos por moluscos nos quais não terão condições de se desenvolver.

É interessante assinalar que $B$. straminea atraiu fortemente miracídios de $S$. mansoni de linhagem mantida na natureza, por outra espécie de vetor. Brasio e col. ${ }^{1}$ (1985) observaram haver certo grau de especificidade na atração miraxonal exercida por moluscos $B$. tenagophila sobre miracídios de S. mansoni da linhagem SJ.

Quanto ao efeito iluminação, Brasio e col. ${ }^{1}$ (1985) verificaram que miracídios da linhagem BH de $S$. mansoni apresentaram maior atração pelos moluscos vetores na presença da luz, e que miracídios da linhagem SJ apresentaram maior atividade na penumbra. No presente experimento não houve efeito da iluminação.

$O$ fato de ter sido observado um exemplar de $B$. straminea com efeito refratário sobre miracidios de $S$. mansoni, sugere que este exemplar deveria conter, em sua hemolinfa ou em suas secreções, substâncias repulsivas aos miracídios. Pode-se especular que, além do componente genético condicionante da susceptibilidade à infecção (Newton ${ }^{7}$, 1953; Richards e col. ${ }^{9}, 1972$ ), os moluscos poderiam possuir também outros fatores, como a emanação de substâncias repulsivas aos miracídios que dificultariam ou impediriam sua infecção.

Houve atração miraxonal exercida por $B$. straminea, Lymnaea columella e Physa sp e pelas $\mathrm{SCW}$ destes moluscos. Physa sp atraiu mais do que Lymnaea columella quando estes moluscos foram utilizados no mesmo experimento, em câmaras diferentes. B. straminea atraiu mais do que $L$. columella. Não houve diferença significativa entre a atração miraxonal exercida por $B$. straminea e Physa sp. Não houve efeito de iluminação.

MAGALHÃES, L.A. et al. [The miraxonal attraction exercised by Biomphalaria straminea, Lymnaea columella and Physa sp over miracidia of Schistosoma mansoni]. Rev. Saúde públ., S. Paulo, 25: 23-7, 1991. The attraction exercised over Schistosoma mansoni miracidia by Biomphalaria straminea, Lymnaea columella and Physa sp using a specially designed apparatus consisting of two circular glass chambers joined by an open channel were studied. The molluscs or their snail-conditioned water (SCW) was placed in one of the chambers (randomly chosen). In the channel ten miracidia was deposited and the count of miracidia in each of the three compartments (the two chambers and the channel) was recorded during fifteen minutes. Ten replications of each experiment with different specimens of molluscs and miracidia were made. Statistical and ad-hoc exploratory data analysis showed that: a) the three species of molluscs and their SCW attract the miracidia; b) Physa sp attract more miracidia 
then $L$. columella. There was an isolated single $B$. straminea specimen that had a repulsive effect to the miracidia.

Keywords: Biomphalaria, physiology. Lymnaea, physiology. Schistosoma mansoni, physiology. Motor activity.

\section{Referências Bibliográficas}

1. BRASTO, B.C.; MAGALHÃES, L.A.; MILLER, J.; CARVALHO, J.F. Atração de miracídios de Schistosoma mansoni por hospedeiros invertebrados: comportamento de miracídios frente a girinos de Hyla fus. covaria. Rev. Saúde públ., S. Paulo, 19: 18-27, 1985.

2. CHERNIN, E. Penetrative activity of Schistosoma mansoni miracidia estimulated by exposure to snail conditioned water. J. Parasit., 58: 209-12, 1972.

3. COX, P.F. The analysis of binary data. London, Methuen, 1970.

4. KLOETZEL, K. Observações sobre o tropismo do mi- racídio do Schistosoma mansoni pelo molusco Australorbis glabratus. Rev. bras. Biol., 18: 223-32, 1958.

5. KLOETZEL, K. Novas observaçōes sobre o tropismo do miracídio de Schistosoma mansoni pelo molusco Austra. lorbis glabratus. Rev. Inst. Med. trop. S. Paulo, 2: 34146, 1960.

6. ETGES, F.J.; CASTER, O.S.; WEBBE, G. Behavioral and developmental physiology of schistosoma larval as related to their molluscans host. Ann. N.Y. Acad. Sci., 266: 480-96, 1963.

7. NEWTON, W.L. The inheritance of susceptibility to infection with Schistosoma mansoni in Australorbis glabratus. Exper. Parasit., 2: 242-57, 1953.

8. PARAENSE, W.L. \& CORREA, L.R. Sobre a ocorrência de duas raças biológicas do Schistosoma mansoni no Brasil. Cienc. e Cult., 15(3): 245-6, 1963.

9. RICHARDS, C.S. \& MERRIT, J.W. Genetic factors in the susceptibility of juvenile Biomphalaria glabrata to Schistosoma mansoni infection. Amer. J. trop. Med. Hyg., 21: 425-34, 1972.

Recebido para publicação em 12/6/1990 Aprovado para publicaçäo em 8/11/1990 